\title{
Axionic Black Holes and an Aharonov-Bohm Effect for Strings
}

\section{Citation}

Bowick, Mark J., Steven B. Giddings, Jeffrey A. Harvey, Gary T. Horowitz, and Andrew Strominger. 1988. "Axionic Black Holes and an Aharonov-Bohm Effect for Strings." Physical Review Letters 61 (25): 2823-26. https://doi.org/10.1103/physrevlett.61.2823.

\section{Permanent link}

http://nrs.harvard.edu/urn-3:HUL.InstRepos:41417265

\section{Terms of Use}

This article was downloaded from Harvard University's DASH repository, and is made available under the terms and conditions applicable to Other Posted Material, as set forth at http:// nrs.harvard.edu/urn-3:HUL.InstRepos:dash.current.terms-of-use\#LAA

\section{Share Your Story}

The Harvard community has made this article openly available.

Please share how this access benefits you. Submit a story.

Accessibility 


\title{
PHYSICAL REVIEW LETTERS
}

\begin{tabular}{lcc}
\hline \hline Volume 61 & 19 DECEMBER 1988 & Number 25 \\
\hline \hline
\end{tabular}

Axionic Black Holes and an Aharonov-Bohm Effect for Strings

\author{
Mark J. Bowick \\ Physics Department, Syracuse University, Syracuse, New York 13244-1130 \\ Steven B. Giddings \\ Lyman Laboratory of Physics, Harvard University, Cambridge, Massachusetts 02138 \\ Jeffrey A. Harvey \\ Joseph Henry Laboratories, Princeton University, Princeton, New Jersey 08544 \\ Gary T. Horowitz and Andrew Strominger \\ Department of Physics, University of California, Santa Barbara, California 93106 \\ (Received 15 September 1988)
}

\begin{abstract}
It is shown that a black hole of fixed mass can carry arbitrary axionic charge. The unique static black-hole solution is found to have vanishing axion field strength but nonvanishing potential. The axion charge cannot be detected by point particles, but can be detected by strings in a process analogous to the Aharonov-Bohm effect. It is argued that the existence of axion charge may play a significant role in the late stages of black-hole evaporation.
\end{abstract}

PACS numbers: $97.60 . \mathrm{Lf}, 03.65 . \mathrm{Bz}, 04.60 .+\mathrm{n}, 14.80 . \mathrm{Gt}$

It is well established that the only stationary vacuum black-hole solutions of Einstein's equation are the twoparameter Kerr family labeled by mass $M$ and angular momentum $J .{ }^{1}$ When gravity is coupled to other fields, black holes may carry conserved charges of the system which can be expressed as surface integrals at infinity. These charges should characterize the final state of gravitational collapse. The obvious example is the case of gravity coupled to the electromagnetic field. The conserved charge (associated with electromagnetic gauge invariance) is electric charge.

There is another field to which gravity may be coupled and whose charge can be expressed as a surface integral at infinity. Consider a two-form (Kalb-Ramond) field $B$ whose field strength is the three-form axion field $H=d B$. (Throughout this paper we will take the axion symmetry to be exact. In superstring compactifications there are a variety of potential axion fields and a variety of mechanisms which could break the associated symmetries and give mass to the axions. Our considerations apply to any linear combination of axion fields which remains exactly massless in the low-energy theory.) The action deter- mining the dynamics of $H$ in four dimensions is

$$
S=\frac{1}{16 \pi} \int H \wedge^{*} H=\frac{1}{16 \pi} \int \sqrt{-g} H_{\mu \nu \lambda} H^{\mu \nu \lambda} d^{4} x,
$$

where the asterisk denotes the Hodge dual and we work in natural units with $h=c=G=1$. $H$ satisfies the Bianchi density

$$
d H=0,
$$

and the equation of motion

$$
d^{*} H=0 .
$$

The axion charge inside a two-surface $\Sigma$ is given by

$$
q=\int_{\Sigma} B
$$

Using $H=d B$, this may be written as

$$
q=\int_{V} H
$$

where $V$ is a spatial three-surface with boundary $\Sigma$.

In this paper we demonstrate that there exist black 
holes (stationary solutions to the field equations of the coupled gravitational-axion system) with nonzero axion charge. We also discuss some implications of this result for the late stages of black-hole evaporation and a means for the measurement of this charge using strings.

The Kalb-Ramond field $B$ has $(D-3)(D-2) / 2$ physical degrees of freedom in $D$ space-time dimensions by virtue of the local invariance $B \rightarrow B+d \Lambda$ where $\Lambda$ is a one-form. Thus $B$ has one physical degree of freedom in four space-time dimensions and may also be described by a single scalar field. Explicitly, Eq. (3) implies that the dual of $H$ is given locally by ${ }^{*} H=d \phi$, with $\phi$ a free (i.e., non-self-interacting) massless scalar field. Thus the dual description of the coupled gravitational-axion system is simply gravity coupled to a massless scalar field. In this dual picture the axion charge is

$$
q=\int_{V} H=\int_{V}^{*} d \phi=\int_{V}\left({ }^{(3)} g\right)^{1 / 2} n_{\mu} \nabla^{\mu} \phi d^{3} x
$$

where $n_{\mu}$ is a unit vector normal to $V$.

At first sight it may seem unlikely that black holes can carry axion charge. If one considers a free massless scalar field in the vicinity of a black hole, one expects that the scalar field either radiates to infinity or falls down the black hole. In the case of spherical symmetry this was proved in Ref. 2. Indeed, there are no static blackhole solutions to the Einstein-scalar field equations where $\phi$ is not constant. (This was shown in Refs. 3 and 4 . A simple proof will be given below.) As discussed above, the axion field $H_{\mu \nu \lambda}$ is locally equivalent to a scalar field. Thus one expects $H_{\mu \nu \lambda}$ will likewise either fall down the black hole or radiate to infinity. This turns out to be correct. This does not, however, mean that the black hole cannot carry axion charge since, roughly speaking, $H_{\mu \nu \lambda}$ may be nonzero at the singularity. To be more precise, note first that if $H_{\mu v \lambda}=0$ the axion stress energy vanishes, so the Schwarzschild metric is still an exact solution. The Schwarzschild metric has topology $R^{2} \times S^{2}$ and has a nontrivial generator of the second cohomology group. Let the Kalb-Ramond field $B$ be this generator, normalized so that

$$
\int_{S^{2}} B=q,
$$

for some real number $q$. Explicitly, $B_{\mu v}=q \epsilon_{\mu v} / 4 \pi r^{2}$, where $\epsilon_{\mu \nu}$ is the induced volume form on the two spheres of spherical symmetry in the Schwarzschild solution. (We can, of course, always add to $B$ the exterior derivative of a one-form $\Lambda$. Since this does not change the charge, we will choose a gauge in which $\Lambda$ vanishes.)

Thus we are led to the following solution for a static black hole of mass $M$ and axion charge $q$ :

$$
\begin{aligned}
d s^{2} & =-(1-2 M / r) d t^{2}+\frac{d r^{2}}{1-2 M / r}+r^{2} d \Omega^{2}, \\
B_{\mu v} & =\frac{q \epsilon_{\mu v}}{4 \pi r^{2}} .
\end{aligned}
$$

We will prove a black-hole uniqueness theorem which shows that (8) are the only static axionic black holes. However, we first establish the following:

Proposition.-The only static black-hole solutions to the coupled Einstein-free scalar field equations are Schwarzschild with constant scalar field.

Proof.-Let $V$ be a surface orthogonal to the timelike Killing field $\xi^{\mu}$, with inner boundary at the horizon $S_{0}$. Let ${ }^{(3)} g_{\mu \nu}$ be the induced metric on $V, D_{\mu}$ its covariant derivative, and $\xi^{\mu} \xi_{\mu}=-\lambda^{2}$. Then a static scalar field satisfies

$$
\begin{aligned}
D^{2} \phi & ={ }^{(3)} g^{\mu \nu} \nabla_{\mu} \nabla_{\nu} \phi \\
& =\nabla^{2} \phi+\lambda^{-2} \xi^{\mu} \xi^{v} \nabla_{\mu} \nabla_{\nu} \phi \\
& =-\lambda^{-2}\left(\xi^{\mu} \nabla_{\mu} \xi^{v}\right) \nabla_{\nu} \phi \\
& =-\lambda^{-1} D^{\mu} \lambda D_{\mu} \phi .
\end{aligned}
$$

Therefore, $D^{\mu}\left(\lambda D_{\mu} \phi\right)=0$. Let $\phi_{0}$ be the constant value of $\phi$ at infinity. Multiplication by $\phi-\phi_{0}$ and integration over $V$ yields

$$
0=\int_{\partial V}\left(\phi-\phi_{0}\right) \lambda D_{\mu} \phi d S^{\mu}-\int_{V} \lambda D^{\mu} \phi D_{\mu} \phi d V .
$$

The boundary of $V$ has two components. At the horizon, the surface integral vanishes since $\lambda=0$. (We are using the fact that $\phi$ is finite on the horizon which is a consequence of the regularity of the space-time geometry. ${ }^{4}$ It is interesting to note that for a conformally coupled scalar field there exist black-hole solutions in which $\phi$ diverges at the horizon but the geometry remains nonsingular. $^{5}$ Unlike the axionic black holes considered here, the conformal scalar black holes are unstable. ${ }^{6}$ ) At infinity, it vanishes since $\phi-\phi_{0}=0$. Since the volume integral is manifestly nonnegative, we conclude that $\phi$ is constant everywhere on $V$. Since $\phi$ is constant, the vacuum black-hole uniqueness theorem of Israel $^{7}$ completes the proof.

We can now prove the following theorem.

Uniqueness theorem for axionic black holes.-The only static solutions to the Einstein-axion field equations which have a regular event horizon, are asymptotically flat, and have finite axion charge $q$ are given by (8).

Proof.-Outside the even horizon, the space-time is topologically $R^{2} \times S^{2}$. Since the first and third cohomology groups vanish, the potential $B$ and $\phi$ are both globally well defined for any axion field $H$. Since the axion field equations are equivalent to the field equations for the massless scalar field $\phi$, one might be tempted to simply invoke the above proposition to conclude that $\phi=0$ and hence $H=0$. This is, however, a subtlety. Recall that

$$
H={ }^{*} d \phi .
$$

A static axion field is one for which the Lie derivative of $H$ with respect to the timelike Killing field is zero. It 
then follows from (11) that $\dot{\phi} \equiv \mathcal{L}_{\xi} \phi$ is constant. So a static axion field does not necessarily correspond to a time-independent $\phi$. However, if $\dot{\phi}$ is nonzero, then $\int_{V} \dot{\phi}=\infty$ where $V$ is as in the previous proposition. This contradicts the assumption that the axion charge is finite. Thus $\dot{\phi}=0$ and the above proposition shows that $\phi$ is constant which implies $H=0$. The vacuum black-hole uniqueness theorem then shows that the metric must be Schwarzschild. The axion potential $B$ must satisfy $d B=0$ (since $H=0$ ). If $B=d \Lambda$ for some one-form $\Lambda$, the charge $q$ vanishes. Nonvanishing charge requires that $B$ be an element of the second cohomology group. Q.E.D.

It is clear that one can add axionic charge (in the above manner) to any solution to Einstein's equation, vacuum or not, defined on a manifold $M$ with $H^{2}(M)$ $\neq 0$. In particular, the Reissner-Nordstrom and KerrNewman solutions can have nonzero axion charge. It is also clear that the above uniqueness theorem easily extends from the static to the stationary case to prove uniqueness of these black-hole solutions as well.

A peculiar feature of the above solutions is that the axion charge does not gravitate since the space-time metric is independent of $q$. The topological nature of the above construction might make one fear that the possibility of arbitrarily large $q$ for fixed $M$ is an artifact of the maximally extended Schwarzschild solution. It seems likely, however, that such a solution could arise during collapse from nonsingular initial conditions. Consider spherically symmetric initial data consisting of an ingoing axion field of amplitude $\epsilon$ spread over a volume $v$. By taking $\epsilon$ small and $v$ large one can make the charge $q$ large keeping the total mass $M$ fixed. Under evolution it is plausible that this regular initial data will collapse to form a black hole of the above type.

Now we have established the existence and uniqueness of black holes with an axion charge, we turn to a discussion of their possible physical consequences. At first sight it appears difficult to measure, even in principle, the axion charge of a black hole. The reason for this is that gauge-invariant couplings of matter to axions typically involve only the gauge-invariant field strength $H_{\mu \nu \lambda}$ and $H_{\mu \nu \lambda}=0$ outside the black hole. The obvious exceptions to this are strings. The coupling of axions to strings takes the form ${ }^{8}$

$$
S_{B}=\frac{1}{2} T \int_{\Sigma} d^{2} \sigma \sqrt{h} B_{\mu v}\left(X^{\lambda}(\sigma)\right) \epsilon^{a b} \partial_{a} X^{\mu} \partial_{b} X^{v},
$$

where $T$ is the string tension, $\Sigma$ is the string world sheet, and $h$ is the world-sheet metric. Such a coupling occurs for fundamental strings and may also occur for axionic cosmic strings.

Consider the propagation of a string in the vicinity of an axion-charged black hole. If the string world sheet is the boundary of a three-surface $V$ not intersecting the singularity of the black hole, then by the Poincare lemma we can write $B=d \Lambda$ for a one-form $\Lambda$ defined every- where on $V$. In this case the coupling in (12) becomes a total derivative

$$
S_{B}=\frac{1}{2} T \int_{\Sigma} d^{2} \sigma \sqrt{h} \nabla_{a}\left(\epsilon^{a b} \partial_{b} X^{v} \Lambda_{v}\right),
$$

and the axion charge has no effect on string propagation. It is clear that if the string is to see the axion charge, its world sheet should encircle the hole, i.e., its world sheet should be in the nontrivial second homology class. An example of such a world sheet is shown in Fig. 1. We can therefore measure the charge on the black hole, at least in principle, by creating a pair of strings at $A$, allowing one of them to lasso the hole and the other to avoid the hole, and then interfering them at point $B$. The action for such a process is just $S_{0}+S_{B}$, where $S_{0}$ is the action for the corresponding process with no axion charge on the black hole. Reinstating factors of the Planck mass, $M_{P}$, we find that the axion charge on the black hole leads to a relative change in phase $q T / M_{P}^{2}$ between the strings when they are compared at point $B$. (As discussed in Refs. 9 and 10, there is a quantization condition on the charge flowing through a homologically nontrivial three manifold. However, in the case we consider there are no such manifolds, and so no such condition modifies our argument.) Clearly, such an experiment will be exceedingly difficult to perform.

The analogy between this effect and the AharonovBohm effect should be clear. In the case of the Aharonov-Bohm effect we have a vector potential $A$ satisfying $d A=0$ everywhere outside of a solenoid, i.e., $d A=0$ everywhere on a manifold $M$ of topology $A^{2} \times R^{2}\left(A^{2}\right.$ is the annulus). The only way to measure the magnetic flux in the solenoid from the outside is to do an interfer-

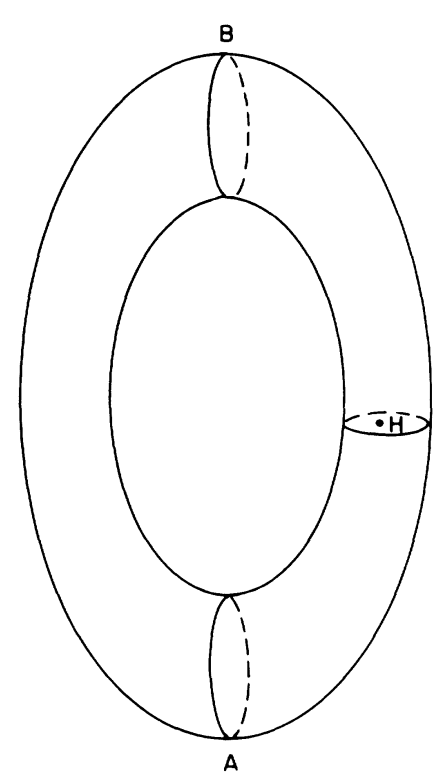

FIG. 1. Illustration of a world sheet that encircles a black hole $H$. The projection of the world sheet onto a spacelike surface $V$ is shown. 
ence experiment involving a particle path that is nontrivial in $H_{1}(M)$. Likewise, in the case of the axionic black hole we do interference using a string world sheet nontrivial in $\mathrm{H}_{2}(M)$.

The solutions considered here have other interesting consequences. As already noted, one peculiar feature of axion-charged black holes is that a black hole of fixed mass can carry arbitrary positive or negative axion charge. In particular, it is classically possible to have a very small mass black hole with nonzero axion charge. This is in contrast to the situation for, e.g., electric charge. It is therefore impossible to make the electric charge $e$ large relative to the mass $M$ since an unphysical naked singularity is encountered for $e>M$.

This situation may have qualitative implications for the process of Hawking evaporation. The semiclassical calculation of black-hole radiation is unaffected by the axion charge since point particles do not couple to $B$ but only to $H$. (By contrast, electrically charged black holes preferentially radiate particles to neutralize the charge.) Thus a black hole will evaporate as before until its mass is on the order of the Planck mass. At this point it will generically have an axion charge which is very large compared to its mass. String or other quantum gravitational corrections to the evaporation process should then become important and may well be affected by the axion charge. However, macroscopic causality and energy conservation place upper bounds on the amount of axion charge that a finite mass black hole can lose in the form of outgoing radiation in a finite amount of time. Thus a Planck-mass black hole with a large axion charge cannot totally evaporate by the Hawking process. This invalidates the simplest arguments in favor of coherence loss by black holes since they assume that the black hole evaporates completely and leaves no remnant in the final state.

An intriguing possibility is that the axionic instantons discussed in Ref. 11 could play an important role in the late stages of black-hole evaporation. Since wormholes couple to the axion field (the general formula is given in Ref. 11), it is possible that a Planck-mass black hole could evaporate completely with the axion charge being swallowed by the wormhole. ${ }^{12}$

Note added: F. Wilczek has pointed out that, since the electromagnetic Aharonov-Bohm effect persists in the presence of symmetry breaking, our Aharonov-Bohm effect might also survive Peccei-Quinn symmetry breaking and be relevant in a theory with massive axions. We also note that the axion charge described here could, in principle, be carried by fundamental particles if the singularity in $H$ at the origin is smoothed out in some more fundamental theory such as string theory.

We wish to thank $P$. Candelas, M. Gleiser, and M. Srednicki for discussions. We also wish to thank the Aspen Center for Physics where most of this work was done. M.J.B. was supported in part by DOE Grant No. DE-FG02-85ER40231; S.B.G. was supported in part by NSF Contract No. PHY-87-14654, and by the Harvard Society of Fellows; J.A.H. was supported in part by NSF Contracts No. PHY80-19754, and PHY86-58033, and by the Alfred P. Sloan Foundation; G.T.H. was supported in part by NSF Contract No. PHY85-06686 and by the Alfred P. Sloan Foundation; A.S. was supported in part by DOE Grant No. 8484062-25000-3 and by the Alfred P. Sloan Foundation.

${ }^{1}$ D. C. Robinson, Phys. Rev. Lett. 34, 905 (1975); P. Mazur, J. Phys. A 15, 3173 (1982), and Phys. Lett. 100A, 341 (1984).

${ }^{2}$ D. Christodoulou, Commun. Math. Phys. 109, 613 (1987).

${ }^{3}$ J. E. Chase, Commun. Math. Phys. 19, 276 (1970).

${ }^{4}$ J. Bekenstein, Phys. Rev. D 5, 1239 (1972).

5 J. Bekenstein, Ann. Phys. (N.Y.) 91, 75 (1975); N. Bocharova, K. Bronnikov, and V. Melnikov, Vestn. Mosk. Univ. Fiz. Astron. 6, 706 (1970).

${ }^{6}$ K. Bronnikov and K. Kireyev, Phys. Lett. 67A, 95 (1978).

${ }^{7}$ W. Israel, Phys. Rev. 164, 1776 (1967).

${ }^{8}$ M. Kalb and P. Ramond, Phys. Rev. D 9, 2273 (1974).

${ }^{9}$ R. Rohm and E. Witten, Ann. Phys. (N.Y.) 170, 454 (1986).

${ }^{10}$ C. Teitelboim, Phys. Lett. 167B, 69 (1986).

${ }^{11} \mathrm{~S}$. B. Giddings and A. Strominger, Nucl. Phys. B306, 890 (1988), and Harvard University Report No. HUTP-88/A036 (unpublished); K. Lee, Phys. Rev. Lett. 61, 263 (1988); J. E. Kim, University of Michigan Report No. UM-TH-88-09 (unpublished).

${ }^{12}$ The general features of this scenario were suggested by F. Dyson [Institute for Advanced Study Report, 1975 (unpublished)]. The possibility of a complete semiclassical description of black-hole evaporation was suggested to us by S. Coleman and $\mathrm{S}$. Smirnakis, who are further pursuing this issue. 